\section{RANDOMIZED MULTICENTER STUDY OF NEOADJUVANT CHEMORADIATION THERAPY (CRT) ALONE OR IN COMBINATION WITH PEMBROLIZUMAB IN PATIENTS WITH RESECTABLE OR BORDERLINE RESECTABLE PANCREATIC CANCER}

\begin{abstract}
${ }^{1}$ Osama Rahma*, ${ }^{2}$ Mathew Katz, ${ }^{3}$ Todd Bauer, ${ }^{4}$ Brian Wolpin, ${ }^{5}$ Chee-Chee Stucky, ${ }^{5}$ Tanios Bekaii-Saab, ${ }^{6}$ Rawad Elias, ${ }^{4}$ Andressa Dias-Costa, ${ }^{4}$ Jonathan Nowak, ${ }^{4}$ Lenehan Patrick, ${ }^{3}$ Gina Petroni, ${ }^{4}$ Stephanie Dougan, ${ }^{3}$ Craig Slingluff. ${ }^{1}$ Dana-Farber Cancer Institute, Boston, MA, USA; ${ }^{2}$ MD Anderson Cancer Center, Houston, TX, USA; ${ }^{3}$ University of Virginia, Charlottesville, VA, USA; ${ }^{4}$ Dana-Farber/Harvard Cancer Center, Boston, MA, USA; ${ }^{5}$ Mayo Clinic, Phoenix, AZ, USA; ${ }^{6}$ Hartford Healthcare Cancer Institute, Hartford, CT, USA
\end{abstract}

Background Pancreatic cancer (PC) is a challenging target for immunotherapy due to its immune-suppressive microenvironment. Neoadjuvant chemoradiation (CRT) can increase the presence of tumor-infiltrating lymphocytes (TILs). We hypothesized that the combination of CRT and pembrolizumab can further expand and activate TILs.

Methods Patients with resectable or borderline resectable PC were randomized 2:1 to the investigational treatment (Arm A) of pembrolizumab $200 \mathrm{mg}$ IV every 3 weeks concurrently with CRT (capecitabine $825 \mathrm{mg} / \mathrm{m} 2$ orally twice daily and radiation 50.4 Gy in 28 fractions over 28 days) or CRT only (Arm B) prior to surgical resection. The primary endpoints were safety and difference in TILs density between Arm A and B assessed using multiplexed immunofluorescence on resected tumor specimens. As a correlate analysis, single cell RNA-sequencing (scRNA-seq) was performed to quantify gene expression in $\mathrm{T}$ cells from tumors and peripheral blood, and to track expanded $T$ cell clonotypes in these compartments $(n=4$ patients Arm A; $n=3$ patients Arm B). The study was amended after enrollment of 37 patients to allow FOLFIRINOX prior to CRT, given changes in standard of care.

Results 37 patients were enrolled (24 Arm A, 13 Arm B). After neoadjuvant therapy, 13 patients had unresectable disease (9 on A, 4 on B), and 24 patients underwent surgery and were evaluable for the TILs primary endpoint (17 arm A, 7 arm B). The mean difference (A-B) in CD8 + T cell density was 36 cells $/ \mathrm{mm} 2$ (95\% CI -85 to 157 , stdev 130) (p 0.48). Additional analysis did not show significant differences in activated cytotoxic $\mathrm{T}$ cells, regulatory $\mathrm{T}$ cells, $\mathrm{M} 1$ - or M2-like polarized macrophages, or granulocytes. The median recurrence free survival (RFS) was 18.2 months on Arm A and 14.1 on Arm B (p 0.41). Overall survival was 27.8 months on Arm A and 24.3 on Arm B (p 0.68) with a median follow up of 2.2 years. The most common grade 3 treatment-related toxicities were lymphopenia reported in $29 \%$ on Arm A and $31 \%$ on Arm B, respectively followed by diarrhea in $8 \%$ on Arm A attributed to CRT. scRNA-seq revealed clonal expansion and expression of co-inhibitory markers among TIL subsets.

Conclusions The combination of CRT and pembrolizumab is safe. Preliminary analysis shows that the addition of pembrolizumab to CRT has minimal effects on intratumoral densities of TILs and other immune cell populations. Single cell transcriptome analyses enable in-depth characterization of the functional responses of $\mathrm{T}$ cells to pembrolizumab in the setting of CRT.

Acknowledgements This study was funded by Merck

Trial Registration NCT02305186

Ethics Approval The study was conducted at 6 sites: University of Virginia, Dana Farber Cancer Institute, MD Anderson Cancer Center (MDACC), Mayo Clinic, Hartford Healthcare Cancer Center, and University of Miami. Written informed consent was provided by the study participants and the protocol was approved by the relevant local IRBs in each site.

http://dx.doi.org/10.1136/jitc-2021-SITC2021.960 